\title{
Separation of specific fractions of synaptosomes by affinity chromatography
}

\author{
L. M. García-Segura, R. Martínez-Rodríguez and R. Martínez-Murillo \\ Laboratorio de Histoquímica, Instituto Cajal, C.S.I.C., Velazquez 144, Madrid-6 (Spain), 4 April 1978
}

Summary. The authors describe a new technique for isolation of specific fractions of synaptosomes, on the basis of their surface glycoproteins, by affinity chromatography using lectin-Sepharose columns.

The use of affinity chromatography has made possible the separation of diverse types of cells, including neurons from sympathetic ganglia ${ }^{1}$. In this note, we describe the possibility of separating different types of brain synaptosomes by affinity chromatography using lectins.

Concanavalin A (Con A) and other lectins are capable of binding to the synaptosome surface ${ }^{2-6}$. By means of affinity chromatography using lectins, diverse glycoproteins fractions can be separated from whole brain or synaptic plasma membranes ${ }^{6-10}$. The existence of these synaptic plasma membrane glycoproteins capable of interacting with lectins ${ }^{6,8,10}$ may serve to separate different types of synaptosomes. Histochemical studies have demonstrated that Con A does not bind to all synaptosomes nor to all synapses in sections from nervous system ${ }^{6}$. Therefore, it should be possible in theory to separate the synaptosomes interacting with Con A from these not interacting. This is also possible in practice. The use of a column of Con A coupled to Sepharose-4B is one way to achieve it. The technique used by us is as follows:

A) Isolation of a synaptosome fraction from brain of rat or mouse, as the technique of Morgan et al. ${ }^{11}$. B) The fraction obtained in A is filtered through a column of Sepharose4B-200. C) The filtrate resulting in B is filtered through a column of Sepharose 4B coupled to Con A (purchased from Sigma Chemical Co). The sample is eluted with PBS (phosphate buffer saline: $0.14 \mathrm{M} \mathrm{NaCl}, 2.7 \mathrm{mM} \mathrm{KCl}$, $16.2 \mathrm{mM} \mathrm{Na}_{2} \mathrm{H} \mathrm{PO}_{4}, 1.5 \mathrm{mM} \mathrm{KH} \mathrm{PO}_{4}$ ) pH 7.4. In this way, the fraction I of synaptosomes not-interacting with Con $\mathrm{A}$ is obtained. The fraction retained by the column is eluted with $0.25 \mathrm{M}$ alpha-methyl-glucoside. This fraction is collected and constitues the fraction II of synaptosomes interacting with Con A. The fraction is centrifugated $(11.500 \times \mathrm{g}, 25 \mathrm{~min})$ and the pellet resuspended in PBS. This is repeated twice or thrice more. After washed in this way with PBS, the synaptosomes are able of interacting again with a column of Con A coupled to Sepharose, which shows that the alpha-methyl-glucoside has been removed. The washed synaptosomes can be liophilized and kept for further studies. The purity of the fractions obtained depends on the purity of the fraction obtained in A. It is also possible to start from whole brain homogenates and so the step $A$ is eliminated. In this case we obtained highly contaminated fractions but useful for morphological studies. Purity of the fractions was controlled by electron microscopy.

The technique described here can be carried out with other lectins, and so it is possible to obtain diverse fractions of synaptosomes on the basis of their predominant membrane glycoproteins. This technique can be used for synaptosomes as well as for other subcellular fractions, such as nuclei which have Con A receptors at membrane level ${ }^{6,12}$.

Affinity chromatography may be a useful technique to separate specific synaptosomes, not only because of their capacity of interacting with lectins, but also due to the presence of another type of surface antigens. Perhaps in this way, the synaptosomes might be separated by the function of their different synaptic receptors.

1 D.J. Dvorak, E. Gipps and Ch. Kidson, Nature 271, 564 (1978).

2 A. Matus, S. DePetris and M. C. Raff, Nature 244, 278 (1973).

3 H. Bittiger and H.P. Schnebli, Nature 249, 370 (1974).

4 C.W. Cotman and D. Taylor, J. Cell Biol. 62, 236 (1974).

5 P. Kelly, C. W. Cotman, C. Gentry and G. L. Nicolson, J. Cell Biol. 71, 487 (1976).

6 L.M. Garcia-Segura, R. Martínez-Rodríguez, R. MartínezMurillo, E. Bogonez and A. Toledano, Acta histochim. 61, 89 (1978).

7 J.P. Susz, H.I. Hof and D.G. Brunngraber, FEBS Lett. 32, 289 (1973).

8 G. Gombos, A. Reeber, J.P. Zanetta and G. Vincendon, Colloque International du CNRS sur les glycoconjugués. p. 829. Editions du CNRS, Paris 1974.

9 J.W. Gurd and H. R. Mahler, Biochemistry 13, 5193 (1974).

10 J.P. Zanetta, I. G. Morgan and G. Gombos, Brain Res. 83, 337 (1975)

11 I.G. Morgan, L.S. Wolfe, P. Mandel and G. Gombos, Biochim. biophys. Acta 241, 737 (1971).

12 J.G. Wood, B.J. McLaughlin and R.P. Barber, J. Cell Biol. 63, 541 (1974).

\section{Factors influencing the distribution of 99m-technetium methylene diphosphonate in bone and soft tissues}

\author{
Ralph A. A. Khan \\ Radiotherapy Department, Supervoltage Unit, Hammersmith Hospital, Ducane Road, London W12 OHS (England), 24 April \\ 1978
}

Summary. The influences of dilution, storage and $\mathrm{pH}$ on the distribution of $99 \mathrm{~m}$-technetium methylene diphosphonate (99m-Tc-MDP) in bone and various soft tissues have been investigated in the rat. The results show a marked departure from normality when large dilution factors and very acidic or basic preparations are used.

The preparation of a stannous methylene diphosphonate kit (Sn-MDP) and its subsequent labelling with $99 \mathrm{~m}$ technetium $(99 \mathrm{~m}-\mathrm{Tc})$ has previously been described ${ }^{1}$. This agent is selectively taken up by the hydroxyapatite crystal of bone, and it has been shown to clear very rapidly from blood and soft tissues ${ }^{1,2}$. It is the most suitable of all the bone scanning agents available because of its high in vitro and in vivo stability, coupled with its superior biological properties.

These experiments were conducted in order to investigate the flexibility of use which a lyophilized Sn-MDP kit provides. The effects on bone mineral uptake of dilution, 